\title{
A daily power generation optimized operation method of hydropower stations with the navigation demands considered
}

\author{
Tianlong Jia ${ }^{1}$, Jianzhong Zhou ${ }^{1, a}$, and Xiaoshen Liu ${ }^{1}$ \\ ${ }^{1}$ School of Hydropower and Information Engineering, Hubei Key Laboratory of Digital Valley Scienceand Technology; Huazhong University \\ of Science and Technology, Wuhan 430074, China
}

\begin{abstract}
Under the influence of hydraulic uncertainty, the various operational safety risks exist in the shortterm hydropower generation scheduling and navigation inevitably. Therefore, it is very crucial to establish a daily power generation optimized method of hydropower stations with the navigation demands considered. The daily power generation optimized operation method was established for generation scheduling and navigation problems of Xiangjiaba hydropower station during the dry seasons. Taking the mutual feedback relationship between the Hydraulic coupling of power generation and navigation as the pointcuts, an improved genetic algorithm was put forward with maximizing daily power generation. A two-dimensional flow model was carried out to validate constraints since the complex flow field was located at the entrance area of the approach channel. With the help of the software MIKE21 FM, the flow field data, such as the water surface lines, flow velocity and direction, was obtained by the numerical simulation. The results showed that the daily power generation optimization and numerical simulation methods could achieve the maximized daily capacity of hydropower station under the premise that ensuring the safety of navigation.
\end{abstract}

\section{Introduction}

Solving the impact of the unsteady flow generated by the daily peaking process of the Xiangjiaba hydropower station on the navigation to the downstream of the dam is the core task of ensuring the navigation safety downstream of the dam[1]. Xiangjiaba Hydropower Station is located in the Jinsha River Gorge at the junction of Yibin County of Sichuan Province and Shuifu County of Yunnan Province, with a controlled drainage area of 458,800 $\mathrm{km}^{2}$ accounting for $97 \%$ of the Jinsha River Basin. The design and development of Xiangjiaba Reservoir is mainly based on power generation, while improving shipping conditions, taking into account flood control and irrigation, and has the effect of counter-regulating the Xiluodu Hydropower Station[2]. The normal water storage level of the reservoir is $380 \mathrm{~m}$, the dead water level is $370 \mathrm{~m}$, the storage capacity is 903 million $\mathrm{m}^{3}$, and it has seasonal adjustment performance. The flood control limit water level in flood season is $370 \mathrm{~m}$, and the flood control capacity is 903 million $\mathrm{m}^{3}[3]$.

Genetic algorithm is an important branch of evolutionary algorithm and belongs to the field of computational intelligence[4]. It has the characteristics of parallel computing and the ability of adaptive search. It can achieve global or quasi-global optimization from multiple initial points and multipath search, and it takes up less computer memory. It is especially suitable for solving large-scale and complex multi-dimensional nonlinear optimization problems.

This paper combines the short-term power generation optimization scheduling model of Xiangjiaba Hydropower and discusses the implementation steps of the genetic algorithm. Due to the complicated flow field in the entrance area of the navigation channel downstream of Xiangjiaba, this paper uses MIKE21 FM to verify the numerical simulation of the navigation channel gate area, and obtain detailed flow field data such as water surface line, flow velocity and flow direction near the gate area.

\section{The maximum power generation model considering the navigation demands}

Effectively balancing the contradictory relationship between hydropower station operation mode and downstream shipping requirements and its efficient solution is the key to establishing a daily power generation plan for power plants that takes into account downstream shipping requirements during

\footnotetext{
a Corresponding author: 2533433765@qq.com
} 
dry season. The output of Xiangjiaba Hydropower Station is affected by the uncertainty of the incoming water during different dispatch periods during the year. In the non-flood period, the hydropower station generally undertakes the peaking task of the power system. In order to ensure the safety and stability of the power grid and the efficient operation of the hydropower station, the hydropower station The output should be consistent with the power system load curve[2]. To this end, fully consider the requirements of the power generation side and the demand side, and according to the shape of the load curve and the power generation capacity allocation schedule of the power station, the power output of the power station will be maximized under the premise of taking into account the downstream shipping.

\subsection{Objective function}

$$
E=\operatorname{Max} \sum_{t=1}^{T} N_{\mathrm{t}}\left(Q_{\mathrm{t}}, H_{\mathrm{t}}\right) \cdot \Delta T
$$

Where $\Delta T$ represents the length of time in each period; $T$ represents the total number of periods in the scheduling period; $E$ represents the total power generation of the hydropower station during the scheduling period; $N_{t}$ represents the output of the hydropower station in the $t$ period, where $Q_{t}, H_{t}$ represent The flow rate and head of the hydropower station during the time period $t$.

\subsection{Constraints}

(1) Reservoir water level constraint

$$
\begin{gathered}
Z_{\min } \leq Z_{t} \leq Z_{\max } \\
\Delta D Z \leq D Z_{\text {max }} \\
\Delta H Z_{t} \leq H Z_{\text {max }}
\end{gathered}
$$

Where $Z_{\mathrm{t}}$ is the water level of the water station during the time period $t, Z_{\min }$ is the lowest reservoir water level of the water level station; $Z_{\max }$ is the highest reservoir water level of the water level station; $\triangle D Z$ is the water level station in the dispatching cycle to get the reservoir water level variation; $D Z_{\max }$ is the maximum allowable amplitude of the reservoir water level in the water level station during the dispatch period; $\Delta H Z_{\mathrm{t}}$ is the water level variation of the reservoir in the water level station within a time period $t, H Z_{\max }$ is the maximum allowable amplitude of the water level of the reservoir in the water level station within a time period $t$.

(2) Reservoir storage constraints

$$
V_{\min } \leq V_{t} \leq V_{\max }
$$

(3) Power station head constraint

$$
H_{\text {min }} \leq H_{t} \leq H_{\text {max }}
$$

(4) Power station outbound flow constraint

$$
Q C_{\min } \leq Q C_{\mathrm{t}} \leq Q C_{\max }
$$

(5) Power plant output constraint

$$
N_{\min } \leq N_{\mathrm{t}} \leq N_{\max }
$$

(6) Stand-alone output and flow constraints

$$
\begin{gathered}
P_{\mathrm{j}}^{\text {min }} \leq P_{\mathrm{j}}(\mathrm{t}) \leq P_{\mathrm{j}}^{\text {max }} \\
Q_{\mathrm{j}}^{\text {min }} \leq Q_{\mathrm{j}}(\mathrm{t}) \leq Q_{\mathrm{j}}^{\text {max }}
\end{gathered}
$$

(7) Water balance constraint

$$
V_{\mathrm{t}+1}=V_{\mathrm{t}}+\left(Q R_{\mathrm{t}}-Q C_{\mathrm{t}}\right) * \Delta t
$$

( 8 ) Daily variable amplitude and hourly amplitude constraint of downstream rivers

$$
\Delta z_{\mathrm{d}} \leq z_{\mathrm{d} \text {,max }} \Delta z_{\mathrm{h}} \leq z_{\mathrm{h}, \max }
$$

\subsection{Application steps of genetic algorithm}

Genetic algorithm is an adaptive global optimization probability search algorithm that simulates the genetic and evolutionary processes of living things in the natural environment[5]. In this example, it can be understood as: $\mathrm{n}$

sets of initial water discharge flow sequences $Q_{1,1}, Q_{1,2}, \ldots, Q_{1, T} ; Q_{2,1}, Q_{2,2}, \ldots, Q_{2, T}$ in the operating environment of Xiangjiaba Hydropower Station. $Q_{n, 1}, Q_{n, 2}, \ldots, Q_{n, T}$ are subject to the above-mentioned model constraints, and their advantages and disadvantages are evaluated by the objective function. Fig. 1 shows a schematic diagram of the operation process of the genetic algorithm.

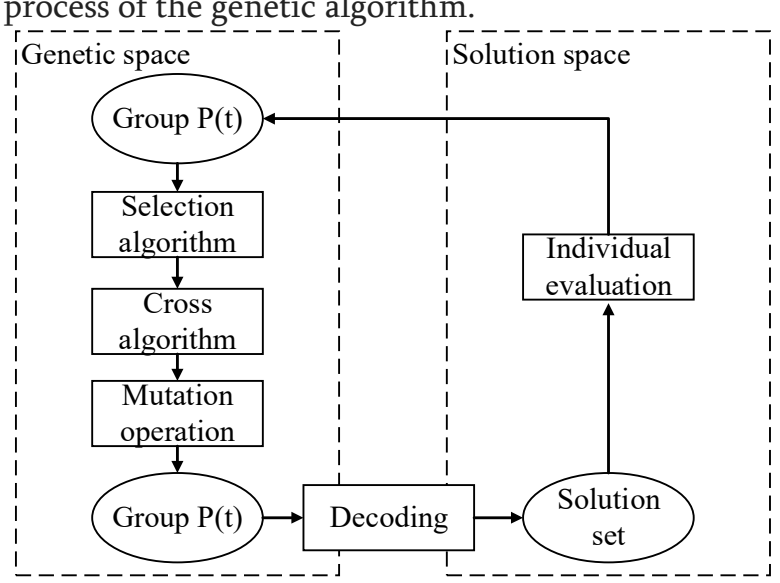

Figure 1. The operation process of genetic algorithm

Genetic algorithm is a kind of stochastic optimization algorithm. It evaluates the chromosomes in the population and manipulates the genes in the chromosomes, and effectively uses the existing information to correct the direction of evolution, and finally converges to the "most adaptable to the environment" individuals. Converging to the global optimal solution, the main steps are:

Step1:Determine the operational parameters of the genetic algorithm.

Step2. Determine the decision variables and constraints.

Equations (2) $\sim(12)$ have given the decision variables and constraints for this problem. 
Step3: Establish an optimization model.

A mathematical model of this problem has been given by equation (1).

Step4: Determine the encoding method.

Because the short-term optimal scheduling of hydropower systems is a multi-dimensional and multi-linear optimization problem with complex constraints, floating-point coding is used.

Step 5: Determine the individual evaluation method.

It can be known from equation (1) that the value range of the objective function is always non-negative, and the optimization goal is to find the maximum value of the function, so the individual fitness $F(Q)$ can be directly taken as the corresponding objective function value plus penalty. Function, which transforms the constrained optimization problem into an unconstrained optimization problem with the penalty function as the objective function.

Step6: Randomly generate the initial population $Q(0)$.

Step7: Select operation.

$n$ the initial individual group, the number of individual individuals copied into the next generation group is determined according to the size of the fitness $F(Q)$. The specific operation process is to calculate the fitness of all individuals in the group $\sum F i(Q)$; secondly, calculate the relative fitness of each individual, $F i(Q) / \sum F i(Q)$, which is for each individual. The probability of inheriting into the next generation group, each probability value constitutes a region, and the sum of all probability values is 1 ; finally, a random number between 0 and 1 is generated, according to which probability region the random number appears in. Determine the number of times each individual is selected.

Step8: Crossover operation.

The individuals resulting from the selection operation are cross-operated to create a new parent individual. For individuals using floating-point numbers, the crossover operation often uses a uniform arithmetic intersection, ie, the child generation is produced by a linear combination of the parent individuals. Suppose that the two parents to be crossed in the $t$-th generation group are: $S t(u)=(u 1, u 2, \ldots, u n)$, $S t(v)=(v 1, v 2, \ldots, v n)$, then cross The resulting children are:

$$
\begin{aligned}
& S t+1(v)=\alpha S t(v)+(1-\alpha) S t(u) \\
& S t+1(u)=\alpha S t(u)+(1-\alpha) S t(v)
\end{aligned}
$$

Where $\alpha$ is a constant between 0 and 1 .

Step9: Variation operation.

Mutation is an effective operator to increase and maintain the diversity of the group, and it is also an effective means to jump out of the local extremum and overcome the "premature convergence". In this paper, non-uniform variation is used to perform mutation operations on individuals in a population.

Step 10: Convergence criterion decision.

For this example, the convergence criteria are:

$$
\begin{gathered}
\left|\frac{Q(t+1)-Q(t)}{Q(T)}\right|<\varepsilon \\
\varepsilon=0.01
\end{gathered}
$$

Convergence criterion determination is an important step to ensure the normal operation of the program and obtain the optimal solution. After Step7 $\sim$ Step9, a new generation group is generated. Applying equation (13) as the convergence criterion, if the convergence criterion is met, the best individual is output and converted. The optimal solution for real numbers. If not, turn to Step7 for the next round of genetic evolution search.

\section{Two-dimensional simulation}

\subsection{General situation of downstream project of Xiangjiaba}

The Xiangjiaba hydropower station which is located in Shuifu county (right bank) of Yunnan province and Yibin county (left bank) of Sichuan province is the last hydropower station of the Jinsha River.The xiangjiaba hydropower station mainly generates electricity, and simultaneously improves navigation conditions, prevents or controls flood, irrigates, controls soil erosion and reverses adjustment for Xiluodu hydropower station.

The Xiangjiaba separates the approach channel from the main channel through the water-dividing dike to ensure the stability of water flow in the approach channel.

The water-dividing dike which is about $793 \mathrm{~m}$ long, is composed of three parts. The upper is $380 \mathrm{~m}$ long, from the tail-bay along the channel direction; The middle is a $167 \mathrm{~m}$ long arc whose radius is $320 \mathrm{~m}$, degree is 30 ; The lower part is $240 \mathrm{~m}$ long, tangent to the arc section and parallel to the direction of the river. The width of the channel at the end of the approach channel is $60 \mathrm{~m}$, and its layout is shown in Fig. 2. 


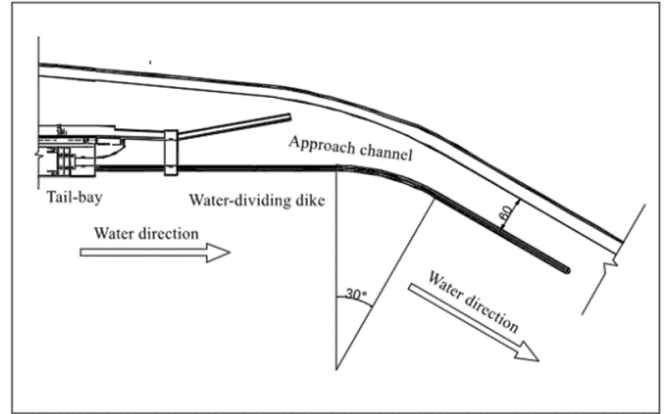

Figure 2. The layout of approach channel

\subsection{Principle of mathematical model}

\subsubsection{Governing equations}

Mike 21FM two-dimensional planar flow model which is based on the constant fluid density, incompressible characteristics and Navier-Stokes equations [6] does not consider coriolis force, waves, wind forces, evaporation, rainfall, etc. Its governing equation is as follows:

$$
\begin{gathered}
\frac{\partial h}{\partial t}+\frac{\partial h u}{\partial x}+\frac{\partial h v}{\partial y}=0 \\
\frac{\partial u}{\partial t}+u \frac{\partial u}{\partial x}+v \frac{\partial u}{\partial y}=f v-g \frac{\partial \eta}{\partial x}-g S_{f, x}+ \\
\frac{1}{h} \frac{\partial}{\partial x}\left(v_{t} h \frac{\partial u}{\partial x}\right)+\frac{1}{h} \frac{\partial}{\partial y}\left(v_{t} h \frac{\partial u}{\partial y}\right) \\
\frac{\partial v}{\partial t}+u \frac{\partial v}{\partial x}+v \frac{\partial v}{\partial y}=f u-g \frac{\partial \eta}{\partial y}-g S_{f, y}+ \\
\frac{1}{h} \frac{\partial}{\partial x}\left(v_{t} h \frac{\partial v}{\partial x}\right)+\frac{1}{h} \frac{\partial}{\partial y}\left(v_{t} h \frac{\partial y}{\partial y}\right)
\end{gathered}
$$

Where $u(x, y, t)$ and $v(x, y, t)$ are the velocity components in the horizontal $x$ and $y$ directions respectively; $h$ denotes the depth of water; $\eta(x, y, t)$ represents the water surface elevation; $g$ is the gravitational acceleration, $f$ is the Coriolis parameter and $v_{t}$ represents the coefficients of horizontal eddy viscosity in the momentum equation; $S_{f, x}$ and $S_{f, y}$ are bed resistance in the $x$ and $y$ directions respectively, which can be expressed as follows:

$$
\begin{aligned}
& S_{f, x}=\frac{n^{2} u \sqrt{u^{2}+v^{2}}}{h^{4 / 3}} \\
& S_{f, y}=\frac{n^{2} v \sqrt{u^{2}+v^{2}}}{h^{4 / 3}}
\end{aligned}
$$

where $n$ is the roughness coefficient.

\subsubsection{Solving method}

To fit the irregular channel boundary, a twodimensional shallow water equations based on unstructured grid was used. The pressure gradient term in the momentum equations was discretized by the semi-implicit method, which eliminates the stability condition constraint caused by the fast surface gravity wave. The convection terms were solved by using the Eulerian-Lagrangian method
(ELM), and the calculated time step was not limited by the stability condition of the Courant number associated with grid scale. The continuity equation was discretized by finite spatial method, which could guarantee conservation of volume water strictly. Finally, the model was characterized by strong boundary adaptability, good numerical stability and high efficiency [7].

\subsection{Numerical simulation}

\subsubsection{Model establishment and mesh generation}

According to the measured sections data of Jinsha River between Xiangjiaba and the downstream boundary and the plane design drawing of the approach channel engineering, the geometric model was established as shown in Fig. 3. The width of the model was $55 \mathrm{~m}$ as the width of the approach channel and the length was $120 \mathrm{~m}$ which is twice the length of the push-train.

Considering the shape characteristics of the entrance area, the unstructured triangular grid was used in the simulation and the grid generation was shown in Fig. 4. In addition, the grid encryption was carried out at the entrance of the approach channel to obtain more accurate local flow field conditions. The total number of model cell grids was 1698 , the number of cell nodes was 1008 , the minimum area of the cell grid was $28.46 \mathrm{~m}^{2}$, and there were 287 grids with an area of less than $40 \mathrm{~m}^{2}$, accounting for $16.9 \%$ of the total grids.
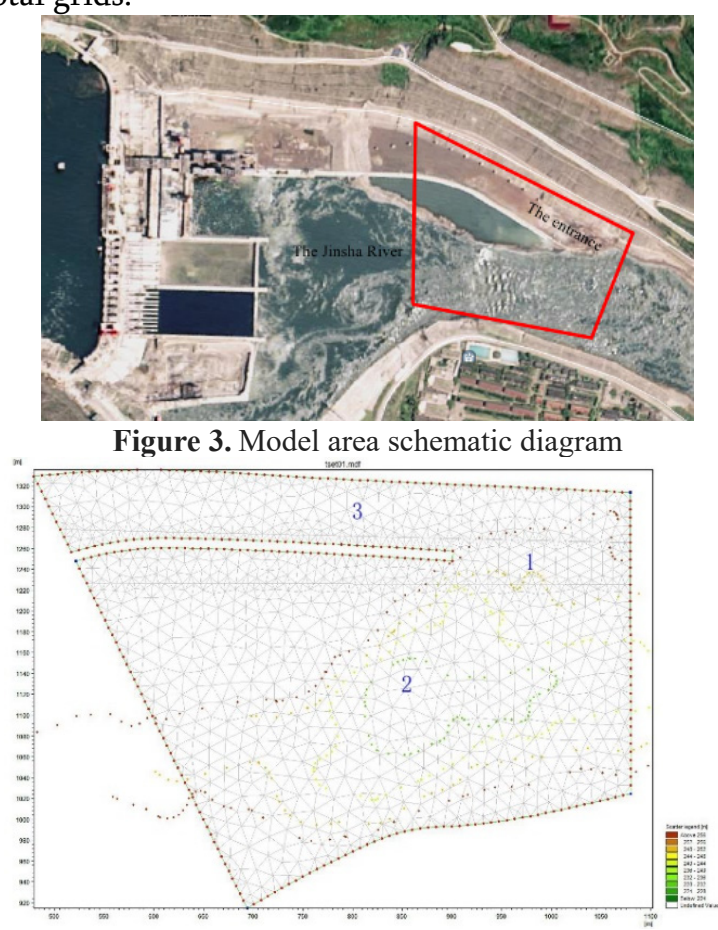

Figure 4. Mesh generation. 


\subsubsection{The boundary conditions}

The upstream inlet of the model was set as the impulse boundary, and the downstream export was set as the water level boundary [6].On the basis of the project design information, the simulated river in this paper was a natural channel, the river channel rough rate was 0.032 , and the eddy viscosity coefficient was 0.28. The influence of tides, precipitation, wind, evaporation, seepage and waves were ignored in the integrated numerical model and the coriolis force was not considered. In order to verify whether the approaching flow of the hydropower generation scheduling meets the requirements of downstream navigation, the simulation period was $24 \mathrm{~h}$ and the time step was $15 \mathrm{~min}$.

\subsubsection{Selection of simulated conditions}

The water level, flow data and the initial downstream water level of the five days' schemes for short-term hydropower generation during the dry seasons were selected for the numerical simulation. The changes of

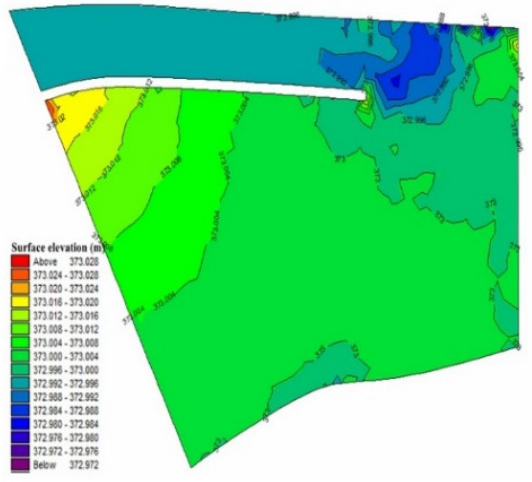

\subsubsection{Calculation results}

In the entrance area of the downstream approach channel, a vertical section was arranged along the axis of the approach channel, which was parallel to the approach channel. The first monitoring point was arranged at the exit boundary of the approach channel, a total of 6 monitoring points, with the distance between the monitoring points at $30 \mathrm{~m}$. The last one was arranged at $150 \mathrm{~m}$ away from the exit boundary towards the downstream and each monitoring point was located at the midpoint of the river bottom.

According to the input conditions of the five days' working conditions, the numerical simulation results were shown in Table 1.

Table 1. Numerical simulation results of five days

\begin{tabular}{|c|c|c|c|}
\hline & $\begin{array}{c}\text { Reflux } \\
\text { condition }\end{array}$ & $\begin{array}{c}\text { Longitudinal } \\
\text { velocity } \\
(\mathrm{m} / \mathrm{s})\end{array}$ & $\begin{array}{c}\text { Transver } \\
\text { se } \\
\text { velocity } \\
(\mathrm{m} / \mathrm{s})\end{array}$ \\
\hline Frist day & $\leq 0.4 \mathrm{~m} / \mathrm{s}$ & $0.42-1.32$ & $0.09-0.48$ \\
\hline
\end{tabular}

flow under five days' conditions were shown in Fig. 5.

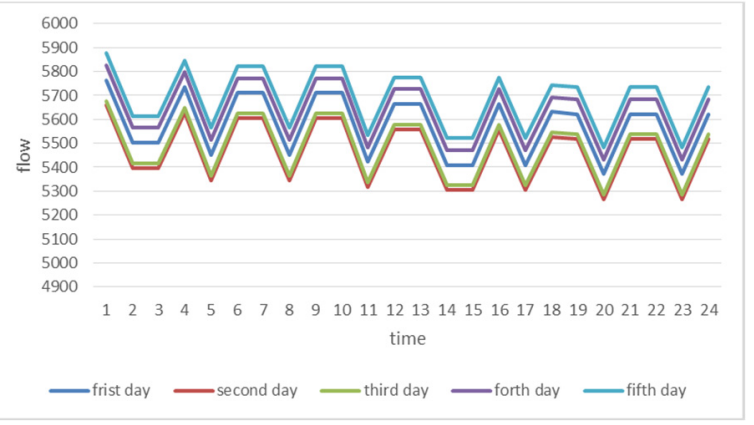

Figure 5. Flow of five days
Figure 6. Flow field analysis

\begin{tabular}{cccc}
$\begin{array}{c}\text { Second } \\
\text { day }\end{array}$ & $\leq 0.4 \mathrm{~m} / \mathrm{s}$ & $0.41-1.30$ & $0.09-0.45$ \\
Third day & $\leq 0.4 \mathrm{~m} / \mathrm{s}$ & $0.41-1.30$ & $0.09-0.47$ \\
Forth day & $\leq 0.4 \mathrm{~m} / \mathrm{s}$ & $0.42-1.34$ & $0.09-0.48$ \\
Fifth day & $\leq 0.4 \mathrm{~m} / \mathrm{s}$ & $0.43-1.35$ & $0.09-0.49$ \\
\hline
\end{tabular}

The isoline of water level, longitudinal velocity and transverse velocity at the entrance area of approach channel on the frist day were shown in Fig .6.

Studies have shown that the maximum longitudinal velocity is $2.7 \mathrm{~m} / \mathrm{s}$, the maximum transverse velocity is $0.45 \mathrm{~m} / \mathrm{s}$ [8], and the maximum reflux velocity is $0.4 \mathrm{~m} / \mathrm{s}$, among the requirements for the flow conditions of the channel.

From analysis results, the longitudinal velocity of five days is less than the maximum limit, and the reflux velocity is less than the maximum limit. However, only the transverse velocity of the second day can meet the maximum transverse velocity, and it of the other days cannot meet the navigation requirements. Therefore, the day scheduling plan of the second day is determined to meet the maximum 
power generation and meet the navigation requirements.

\section{Conclusion}

On the basis of determining the navigation constraints in the downstream of Xiangjiaba hydropower station, a short-term optimal operation method for hydropower station with constraint treatment of hydraulic factors in combination with the twodimensional hydrodynamic model is proposed, which can effectively guarantee the downstream shipping requirements of hydropower station. An improved genetic algorithm is used to find the optimal solution, to obtain the short-term hydropower generation scheduling for maximum power generation. Then, through the hydraulic elements such as flow rate and water level, the connection with the two-dimensional hydraulic model is established, and the navigation requirements of the hydraulic elements are verified in real time. Finally, the scheme of daily optimal operation and navigation of the power station can be obtained.

\section{Acknowledgements}

The work is supported by the National Key $R \& D$

Program of China (No. 2016YFC0401910).

\section{References}

1. Yongqiang Wang,Dewei Mu,Xueming Li,Deyu Zhong and Baosheng Wu. Optimized operation mode of daily power generation in the dry season of Xiangjiaba Hydropower Station with consideration of downstream shipping requirements[J].Journal of Tsinghua University(Science and Technology),2015,55(02) :170-175+183.

2. Kaiyong Liu. Research and application of shortterm power generation plan for cascade hydropower stations [D]. Huazhong University of Science and Technology, 2013.

3. Zhang Rui, Zhang Lisheng, Wang Xuemin, Wang Yongqiang.Multi-objective profit-scheduling scheduling model for cascade reservoirs in the lower reaches of Jinsha River and its application[J].Journal of Sichuan University (Engineering Science Edition),2016,48(04):32$37+53$

4. Wang Xiaoan, Zhou Jianzhong, Wang Hui, Li Chengjun.Research and Application of Genetic Algorithm in Short-Term Power Generation Optimization Scheduling[J].Computer Simulation,2003(10):120-122+128.

5. B. Rajeswari, K. S. Amirthagadeswaran .Study of machinability and parametric optimization of end milling on aluminium hybrid composites using multi-objective genetic algorithm[J]. Journal of the Brazilian Society of Mechanical Sciences and Engineering, 2018, Vol.40 (8), pp.1-15.

6. Luo Ming, Huang Er, Li Gang. Optimization of diversion inlet in irrigation district based on Mike21 FM numerical simulation[J]. International Journal Hydroelectric Energy, 2018(3).

7. Casulli V, Walters R A. An unstructured grid, threedimensional model based on the shallow water equations[J]. International Journal for Numerical Methods in Fluids, 2015, 32(3):331-348.

8. Gong Decheng, Bai Chengliang, Yang Shisheng. Discussion on navigable flow condition of entrance area and connecting section of navigable building $[\mathrm{J}]$. China's water transport, 2007, 7(1):32-33. 